\title{
Nivel de conocimiento sobre los efectos del cambio climático, distrito Bongará, Amazonas, Perú, 2019
}

\section{Level of knowledge about the effects of climate change, Bongará district, Amazonas, Peru, 2019}

\author{
Hilda Panduro Bazán de Lázaro ${ }^{1}$
}

\begin{abstract}
RESUMEN
La presente investigación tuvo como objetivo evaluar el nivel de conocimiento sobre los efectos del cambio climático, en el distrito Bongará, Amazonas, Perú, 2019. La población estuvo constituida por 1800 habitantes del Puente San Jerónimo, Palo Seco, Donce, Nuevo Churuja, Churuja, Nuevo Horizonte, Matiaza Rimachi, Cocahuayco y puente Tingorbamaba determinándose una muestra de 73 pobladores. Los métodos utilizados fueron el inductivo-deductivo y el analítico-sintético. Se aplicó una encuesta para la recolección de la información (pre y post). Se encontró que, en la pre encuesta, solamente el $7 \%$ tuvieron el nivel de conocimiento bueno, mientras que el 32\% obtuvieron el nivel regular y el $62 \%$ el nivel malo. Sin embargo, en la post encuestan se observó que, el 31,5\% obtuvo el nivel conocimiento bueno, el 48\% regular y el malo se redujo al 20,5\%, predominando el nivel regular, ninguno de los encuestados aparece en el nivel excelente. Se concluye que predomina nivel de conocimiento regular sobre los efectos del cambio climático.
\end{abstract}

Palabras claves: nivel de conocimiento - cambio climático

\begin{abstract}
The present research aimed to evaluate the level of knowledge about the effects of climate change, in the Bongará district, Amazonas, Peru, 2019. The population consisted of 1800 inhabitants of the San Jerónimo Bridge, Palo Seco, Donce, Nuevo Churuja, Churuja, Nuevo Horizonte, Matiaza Rimachi, Cocahuayco and Tingorbamaba bridge determining a sample of 73 residents. The methods used were inductive-deductive and analytical-synthetic. A survey was applied to collect the information (pre and post). It was found that in the pre-survey, only $7 \%$ had the good level of knowledge, while 32\% obtained the regular level and $62 \%$ the bad level. However, in the post-survey it was observed that $31.5 \%$ obtained the good knowledge level, $48 \%$ regular and the bad knowledge was reduced to $20.5 \%$, with the regular level predominating, none of the respondents appears at the level Excellent. It is concluded that the prevailing level of regular knowledge about the effects of climate change.
\end{abstract}

Keywords: level of knowledge - climate change. 


\section{INTRODUCCIÓN}

A nivel mundial el principal problema medioambiental que afronta la humanidad como consecuencia de las emisiones permanentes de gases del efecto invernadero, mediante las actividades antropogénicas de los países industrializados y del mal manejo de los recursos naturales como producto de la irresponsabilidad frente al medio ambiente tanto a nivel individual como colectivo, ocasionando el cambio climático, cuyos efectos y consecuencias se ven reflejados en el deterioro del planeta tierra. El Perú es el tercer país más frágil después de Bangladesh y Honduras debido a los fenómenos hidrometereológicos como huaicos, fuertes lluvias sequias, granizadas, inundaciones. El eje de investigación de este informe intenta evadir las posturas y desentrañar la realidad a través de datos que permitan observar el fenómeno desde una mirada objetiva y cuantitativa. Actualmente cada vez cobra mayor impulso en la opinión pública, los medios de comunicación, la política y la comunidad científica la teoría del calentamiento global y sus consecuencias sobre el cambio climático. La comunidad internacional quedó sorprendida como el mandatario de Estados Unidos, Donald Trump, quien afirmó que lo del calentamiento global es un cuento chino e invento de los ambientalistas de izquierda. Esto demuestra que dicho mandatario desconoce el significado del cambio climático, así como de sus causas y consecuencias, este nivel de desconocimiento no es ajena a un buen porcentaje de la población, incluyendo a los sujetos involucrados en esta muestra de esta investigación. Para coadyuvar en el nivel de conocimiento del cambio climático, se presenta los siguientes antecedentes:

Gorrini (2017) en su trabajo "Cambio climático", sostiene que el calentamiento global se define como el fenómeno en el cual se percibe un aumento de la temperatura media global. En los últimos años se ha dado una particularidad en la que se registra un aumento de $1^{\circ} \mathrm{C}$ de la temperatura media mundial desde 1850 hasta la actualidad, hecho que ha ido acrecentándose al paso de los últimos años, donde se detectó un aumento de $0,6^{\circ} \mathrm{C}$ en el período 1970 2000. Este suceso en el que se observa una variación en la temperatura de la Tierra es atribuido por el consenso de la comunidad científica internacional a la acumulación de gases de efecto invernadero en la atmósfera, presuntamente resultantes del uso de combustibles fósiles y otros procesos industriales; como el dióxido de carbono, metano, óxido nitroso y clorofluorocarbonos. Este aumento en la temperatura conlleva a un cambio climático de escala global.

En un principio creíamos en una "naturaleza benigna", esto significa que la naturaleza es un sistema robusto que responde bien a los desórdenes causados por el hombre siempre volviendo de nuevo a su estado natural. Pero desde hace tiempo advertimos una "naturaleza perversa / tolerante", lo que significa básicamente que la naturaleza puede tolerar desordenes hasta cierto punto. Si los desórdenes son pequeños, la naturaleza volverá a su equilibrio. Grandes desordenes suponen una amenaza al funcionamiento de la naturaleza. El clima definitivamente está cambiando, el aumento de la concentración de $\mathrm{CO} 2$ atmosférico durante los últimos dos siglos de industrialización presenta actualmente sus consecuencias; las temperaturas y el nivel de los océanos indican un aumento, los casquetes polares se derriten y los ecosistemas dan indicios de adaptaciones a las nuevas condiciones. $\mathrm{La}$ controversia nace de cara hacia el futuro, en cómo responderá el clima frente a estos cambios, y si la magnitud de los sucesos aumentará afectando poblaciones, sociedades, sistemas de producción, economías y ecosistemas; o si el complejo sistema climático amortiguará los cambios y reducirá las consecuencias. Las teorías y explicaciones se abren hacia los dos caminos, pero más allá de estas se erige el factor más determinante, concreto y predictivo que es la realidad presente. Esta refleja cambios considerables en la Tierra con tan sólo seis décimas de grados centígrados de aumento de la temperatura en los últimos 120 años, y habla de que a medida que se presenten temperaturas más altas la envergadura de las alteraciones será mayor, potenciando a su vez las causas del aumento de la temperatura en un sistema sinérgico. Por mínimos que sean los cambios afectarán los patrones climáticos como las corrientes marinas y los vientos, reubicando las precipitaciones y las zonas cultivables, lo que seguramente afectará a las poblaciones ya asentadas con relación a estas áreas. Hasta el momento no se ha tomado ninguna medida concreta para detener el calentamiento global. El protocolo de Kioto, emblema entre los tratados internacionales para intentar frenar los efectos del calentamiento global, ni siquiera es apoyado por las grandes potencias emisoras de gases de efecto invernadero como EE.UU., China, India, Australia, Rusia, entre otras. Además, su objetivo de reducción de 5,2\% de las emisiones de gases de efecto invernadero es deficiente frente a la afirmación de los científicos de que hay que reducir entre un 50-70\% las emisiones de $\mathrm{CO} 2$ para mantener la concentración en la atmósfera estable. Muchos proponen medidas para lograr un mejor desempeño en los procesos de obtención y utilización de la energía, o utilizar fuentes de energía alternativas no contaminantes, impulsadas por campañas de educación, información, e incentivos y desincentivos 
económicos. Pero la realidad es que no existe un remedio inmediato al problema, y las consecuencias son inevitables. Sólo se puede reducir la magnitud de estas y prepararnos frente a los potenciales cambios. No hay mejor frase que refleje nuestra situación actual frente al futuro respecto del cambio climático que la pronunciada por Winston Churchill en la década de 1930, cuando hubo una gran tormenta que azotó Europa y este apercibió a los británicos que debían prepararse para sucesos similares debido a que el clima iba a cambiar, y cuando nadie le creyó dijo: "La época de dilatarse, de medidas parciales, de recursos calmantes e inesperados, de retrasos, está terminando. En su lugar estamos entrando en un periodo de consecuencias".

Romo (2015) en su tesis titulada "Levantamiento de línea base para la implementación de medidas de adaptación al cambio climático en la comunidad de minas Chupa, Parroquia San José de Minas - Distrito Metropolitano de Quito", entre sus principales conclusiones tenemos: Los principales impactos de los efectos al cambio climático en Minas Chupa son: perdida de cultivos, escasez de agua, pérdidas económicas, menor fertilidad en suelos, daño a viviendas y a la salud de la población. La principal limitante es el factor económico y el apoyo de los gobiernos locales. Los problemas de salud en la población se agravan frente a servicios de salud deficientes. La encuesta revela que los habitantes de Minas Chupa no ven solución frente a los cambios climáticos, la falta de iniciativas propositivas a esta interrogativa nos demuestra que las medidas adaptativas propuestas deberán iniciar de cero y ser acompañadas de un fuerte proceso de educación para reducir la incredibilidad.

Badillo (2018) en su tesis titulada Análisis del cambio climático y su incidencia en el sector agrícola en el Ecuador en el año 2017, llegó a las siguientes conclusiones El cambio climático es producto de las actividades productivas del ser humano y el uso irracional de los recursos, sin planificar el retorno adecuado de los desechos a la naturaleza. Es determinante crear una consciencia social sobre el valor de proteger el medio ambiente. El desarrollo sostenible es posible siempre y cuando se respete el equilibrio entre el medio ambiente y el crecimiento económico.

Chanca (2016) en su tesis "Percepciones y Perspectivas Socioculturales de los pobladores sobre el cambio climático en Huayllahuara - Huancavelica 2015", concluye en lo siguiente: Que las percepciones y perspectivas socioculturales de los pobladores sobre el cambio climático son conocimientos habituales que expresan hechos socioculturales como mitos, ritos y creencias sobrenaturales que se transmiten de generación en generación; en cambio los conocimientos científicos modernos se propagan a través de los medios de comunicación y los centros de estudios, que vienen conllevando al descrédito de los primeros. Las percepciones sobre el cambio climático son diferentes según el saber local y los conocimientos adquiridos por lo que el $50 \%$ manifestó que el fenómeno es malo para la sociedad en su conjunto, más del $40 \%$, no ha sido afectada por desastres naturales de consideración, y cerca del $70 \%$ de encuestados dijeron que el cambio climático nunca trae beneficios a favor de los pobladores, se afirma que los pobladores identifican adecuadamente este fenómeno natural. Asimismo, casi el 60\% señalaron que la destrucción de la capa de ozono es el principal causante del cambio climático, a ello se suma la contaminación del aire, agua y suelo. Se confirmó, también, que el clima ya no es igual que antes: hay débil presencia de lluvias percibida por más del $30 \%$ y prolongadas sequías, hace más calor para más del $80 \%$ y más frío para el $16 \%$ y los vientos soplan más fuerte para cerca del $60 \%$ de encuestados.

Teniendo en cuenta los distritos señalados y el estudio del nivel de conocimiento sobre el cambio climático, se propuso el siguiente problema de investigación: ¿Cuál es el nivel de conocimiento sobre los efectos del cambio climático en el distrito Bongará, Amazonas, Perú, 2019?

Esta investigación se justifica mediante un serio análisis de la realidad ambiental y los efectos que viene ocasionando el cambio climático, así como su comprensión en el conocimiento científico sobre la existencia y el uso del plástico en la zona urbana y rural, así como de sus causas y consecuencias que compromete a la calidad de vida, que tanto se anhela y pregona. Por otro lado, se convierte de vital importancia para la Universidad y su proyección a la comunidad en el marco de la responsabilidad social. En este caso hacia las rutas que une Pedro Ruiz y Chachapoyas, fundamentalmente en la Provincia de Bongará, donde se está proponiendo mitigar los efectos del cambio climático en la vía que comprende tres distritos: Jazán, Churuja y Pablo Valera, principalmente por las poblaciones de : Puente de San Jerónimo, Palo Seco, Donce, Nuevo Churuja, Churuja, Nuevo Horizonte, Matiaza Rimachi y Cocahuayco. Toda esta ruta está alrededor del Río Utcubamba. En este sentido, este estudio fue enmarcado en su gran importancia, porque se propuso un trabajo con el fin de mitigar los efectos del cambio climático mejorando la calidad de vida de los habitantes al promover el desarrollo de capacidades y actitudes ambientales de esos sectores de la ruta, con 
el desarrollo de talleres de sensibilización y concienciación ecológica en los pobladores para reducir la presencia del plástico (cero plásticos), logrando de esta manera un anhelo de los investigadores para beneficio a los habitantes de esta zona y el cuidado sostenible de la flora y fauna, convirtiéndose en una ruta paisajista. Los talleres desarrollados con los pobladores y las coordinaciones permanentes con las autoridades distritales y de las comunidades comprometidas, se logró promover significativamente la conciencia ambiental, así como la elaboración de contenedores para ir reduciendo la contaminación del ambiente, así como reducir al máximo los residuos de plástico, que constantemente arrojan los pasajeros de los vehículos motorizados. Posteriormente se verificó que los objetivos fueron alcanzados en un $79 \%$ en la zona investigada.

\section{MATERIAL Y MÉTODOS}

\subsection{Diseño de Investigación}

Diseño de investigación, se utilizó el diseño transversal correlacional - causal, que permitieron medir y describir las relaciones de las dos variables.

Para el presente estudio, el esquema es el

Siguiente:

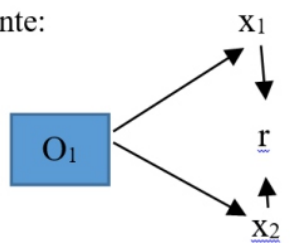

Donde:

O1 = Información de interés

$\mathrm{X} 1$ = Pre encuesta

X2 $=$ Post encuesta

$\mathrm{r}=$ Relación

\subsection{Población, Muestra y Muestreo}

Población: Estuvo conformado por 1800 pobladores del Puente de San Jerónimo, Palo Seco, Donce, Nuevo Churuja, Churuja, Nuevo Horizonte, Matiaza Rimachi, Cocahuayco y puente Tingorbamba.

Muestra: Estuvo determinada por 73 pobladores.

Muestreo: Fue de tipo probabilístico, ya que la muestra se seleccionó aleatoriamente.

\subsection{Fuentes de información}

Municipios Distritales

\author{
Juntas Vecinales \\ Comisarias locales
}

2.4. Métodos, técnicas e instrumentos de recolección de datos y procedimiento

Métodos: Los métodos utilizados fueron:

-Analítico-Sintético

- Inductivo-Deductivo

Técnicas: Observación

Instrumentos: Para la recolección de la información se utilizó una encuesta.

\section{Procedimiento}

$1^{\circ}$. Aplicación de un pre encuesta para diagnosticar en los pobladores, el nivel de conocimiento sobre los efectos del cambio climático.

Desarrollo de charlas y talleres coordinados en fechas y horas con las autoridades.

$3^{\circ}$. Ejecución y participación activa y conjunta en jornadas de limpieza.

$4^{\circ}$. Distribución en los medios de información masiva y en los terminales de transportes de bolsa de tela, afiches, volantes sobre el cuidado del medio ambiente.

$5^{\circ}$. Elaboración de 7 colectores para la comunidad de Nuevo Horizonte y Churuja.

$6^{\circ}$. Instalación de la botella ecológica en la Ruta Jazán, Churuja y Valera.

$7^{\circ}$ Aplicación de la post encuesta a la muestra investigada.

\section{RESULTADOS}

Tabla 1. Nivel de conocimiento sobre los efectos del cambio climático.

\begin{tabular}{|c|c|c|c|c|}
\hline \multirow{3}{*}{$\begin{array}{c}\text { Nivel de } \\
\text { conocimiento }\end{array}$} & \multicolumn{4}{|c|}{ ENCUESTA } \\
\hline & \multicolumn{2}{|c|}{ PRE } & \multicolumn{2}{|c|}{ POST } \\
\hline & f & $\%$ & f & $\%$ \\
\hline Exelente (46-60) & 0 & 0 & 0 & 0 \\
\hline Bueno (31-45) & 05 & 7,0 & 23 & 31,5 \\
\hline Regular (16-30) & 23 & 32,0 & 35 & 48 \\
\hline Malo (00-15) & 45 & 62,0 & 15 & 20,5 \\
\hline TOTAL & 73 & 100 & 73 & 100 \\
\hline
\end{tabular}




\section{DISCUSIÓN}

La presente investigación estuvo orientada a evaluar el nivel de conocimiento sobre los efectos del cambio climático en los pobladores de los distrititos de Jazán, Churuja y Pablo Valera, región Amazonas, y por lo tanto, mitigar al máximo la contaminación del ambiente con plásticos, para darle sostenibilidad ambiental, por lo que entre las investigaciones citadas coincidimos con Gorrini (2017)", quien sostiene que el calentamiento global se define como el fenómeno en el cual se percibe un aumento de la temperatura media global, sustentado así mismo por la comunidad científica internacional al expresar que la acumulación de gases de efecto invernadero en la atmósfera, presuntamente resultantes del uso de combustibles fósiles y otros procesos industriales; como el dióxido de carbono, metano, óxido nitroso y clorofluorocarbonos conlleva a un cambio climático de escala global. De igual manera con la investigación de Romo (2015) quien concluye que, los principales impactos de los efectos al cambio climático en Minas Chupa son: perdida de cultivos, escasez de agua, pérdidas económicas, menor fertilidad en suelos, daño a viviendas y a la salud de la población; la principal limitante es el factor económico y el apoyo de los gobiernos locales; los problemas de salud en la población se agravan frente a servicios de salud deficientes, asimismo, los resultados de nuestra investigación concuerdan con los de Romo al revelar que los habitantes de Minas Chupa no ven solución frente a los cambios climáticos, la falta de iniciativas propositivas a esta problemática nos demuestra que las medidas de solución deben estar sujetos a un fuerte proceso de educación ambiental para reducir la incredibilidad, resultados que guardan similitud con nuestro trabajo, al concluir que el nivel de conocimiento sobre los efectos del cambio climático es regular.

Así mismo, la investigación realizada por Badillo (2018) se asemeja con sus conclusiones al expresar que el cambio climático es producto de las actividades productivas del ser humano y el uso irracional de los recursos, sin planificar el retorno adecuado de los desechos a la naturaleza. Esto implica crear una consciencia social sobre el valor de proteger el medio ambiente. De la misma forma concordamos con las conclusiones arribadas por Chanca (2016) respecto a las percepciones y perspectivas socioculturales de los pobladores sobre el cambio climático como conocimientos habituales que expresan hechos socioculturales, como mitos, ritos y creencias sobrenaturales que se transmiten de generación en generación; contrariamente a los conocimientos científicos modernos que se propagan a través de los medios de comunicación y los centros de estudios, que vienen conllevando al descrédito de los primeros. Asimismo, señalaron que la destrucción de la capa de ozono es el principal causante del cambio climático, a ello se suma la contaminación del aire, agua y suelo. Se confirmó, también, que el clima ya no es igual que antes: hay débil presencia de lluvias y prolongadas sequías, hace más calor y más frío, y los vientos soplan más fuerte para cerca del $60 \%$ de encuestados, investigaciones que guardan relación con nuestros resultados obtenidos, conforme se puede observar en la tabla 1 de la pre encuesta, donde solamente el $7 \%$ de los encuestados, obtuvieron el nivel de conocimiento bueno sobre los efectos del Cambio Climático, mientras que el 32\% tuvieron el nivel regular y el $62 \%$ el nivel malo. Predominando el nivel malo. Sin embargo, en la post encuesta se logró, que el 31,5 \% obtuvo el nivel conocimiento bueno sobre los efectos del Cambio Climático, el $48 \%$ regular y el nivel malo se redujo al $20,5 \%$, predominando el nivel regular. Cabe manifestar que en ninguno de los datos obtenidos aparece el nivel excelente. Los resultados de la post encuesta fueron significativos, teniendo en cuenta que antes de su aplicación, se realizaron actividades para propiciar la sensibilización sobre el significado del cambio climático, elevar el conocimiento en el cuidado al medio ambiente, como parte de los objetivos e hipótesis planteados en la presente investigación.

Finalmente, es necesario tener en cuenta sobre el protocolo de Kioto, emblema entre los tratados internacionales para intenta frenar los efectos del calentamiento global, ni siquiera es apoyado por las grandes potencias emisoras de gases de efecto invernadero como EE.UU., China, India, Australia, Rusia, entre otras. Además, su objetivo de reducción de $5,2 \%$ de las emisiones de gases de efecto invernadero es deficiente frente a la afirmación de los científicos de que hay que reducir entre un $50-70 \%$ las emisiones de $\mathrm{CO} 2$ para mantener la atmósfera estable, a pesar que muchos proponen medidas para lograr un mejor desempeño en los procesos de obtención y utilización de la energía, o utilizar fuentes de energía alternativas no contaminantes, impulsadas por campañas de educación, información, e incentivos económicos, sin embargo en la práctica no existe un remedio inmediato al problema, y las consecuencias son inevitables, por eso tenemos que estar en permanente alerta y en buscar soluciones alternativos inmediatos. Esta situación lo han entendido muy bien nuestros investigados, de allí que siempre comentan y planifican campañas de limpieza y de reciclaje. Incluso con los investigadores hay un compromiso de poner en práctica las $5 R$. 


\section{CONCLUSIONES}

1. El 31,5\% tiene nivel de conocimiento bueno sobre los efectos del cambio climático.

2. El 48\% tiene nivel de conocimiento regular sobre los efectos del cambio climático.

3. El $20.5 \%$ tiene nivel de conocimiento malo sobre los efectos del cambio climático.

\section{REFERENCIAS BIBLIOGRÁFI-CAS}

Aledo, A. (2012). La crisis ambiental y su interpretación sociológica. TYCEA-BLE.

Badillo, M. (2018) Tesis titulada Análisis del cambio climático y su incidencia en el sector agrícola en el Ecuador en el año 2017, para obtener el título de Licenciada multilingüe en negocios y relaciones internacionales. Pontificia Universidad Católica del Ecuador Facultad de Comunicación, Lingüística y Literatura Escuela Multilingüe de Negocios y Relaciones Internacionales. Quito, Ecuador

Chanca, A. (2016) Tesis "Percepciones y Perspectivas Socioculturales de los Pobladores Sobre el Cambio Climático en Huayllahuara - Huancavelica 2015". Para optar el Grado Académico de Doctor en Ciencias Ambientales y Desarrollo Sostenible. Universidad Nacional del Centro del Perú, Escuela de Posgrado, Facultad de Ciencias Forestales y del Ambiente, Huancayo-Perú

Gorrini, F. (2017). Cambio Climático. Buenos Aires, República Argentina). Maravillas Naturales (veoverde@betazeta.com)

Norhhaus, D. y Romer, P.(Martes 9-10-2018). Estudio sobre el cambio climático recibe el Nobel. La República.

Romo, M. (2015), tesis "Levantamiento de línea base para la implementación de medidas de adaptación al cambio climático en la comunidad de minas Chupa, Parroquia San José de Minas - Distrito Metropolitano de Quito". Para obtener el grado de Magister en Gestión Ambiental en la Industria. Universidad Internacional Sek. Facultad de Ciencias Ambientales. Quito, Ecuador. 